\title{
Covariant model for proton-proton bremsstrahlung: Comparison with high-precision data
}

\author{
M. D. Cozma, ${ }^{1}$ G. H. Martinus, ${ }^{1}$ O. Scholten, ${ }^{1}$ R. G. E. Timmermans,${ }^{1}$ and J. A. Tjon ${ }^{1,2}$ \\ ${ }^{1}$ KVI, University of Groningen, Zernikelaan 25, 9747 AA Groningen, The Netherlands \\ ${ }^{2}$ Institute for Theoretical Physics, University of Utrecht, Utrecht, The Netherlands
}

(Received 17 July 2001; published 8 January 2002)

\begin{abstract}
We compare a relativistic covariant model for proton-proton bremsstrahlung with high-quality data from KVI. The agreement in large parts of phase space is satisfactory. However, remarkably large discrepancies are observed for specific kinematic regions. These failures are shown to occur primarily when the final twonucleon system has energies less than about $15 \mathrm{MeV}$.
\end{abstract}

DOI: 10.1103/PhysRevC.65.024001

PACS number(s): 24.10.Jv, 21.30.Cb, 25.20.Lj

\section{INTRODUCTION}

The proton-proton bremsstrahlung $(p p \gamma)$ process has attracted significant attention over the years, both experimentally and theoretically. In recent years, several new experiments have been performed [1-6], inspiring many new theoretical investigations [7-14]. In particular, a number of microscopic models have been developed to describe the $p p \gamma$ process. Examples are the potential model of Nakayama et al. [8] and the covariant model of Martinus et al. [10]. The theoretical predictions of these models could be compared to the $p p \gamma$ cross sections and analyzing powers for the TRIUMF experiment at $280 \mathrm{MeV}$ [1]. The agreement of theory with these TRIUMF data is rather good, especially for the cross sections, provided that the experimental cross sections are renormalized [1]. Some outstanding discrepancies occur, however, for certain asymmetric proton angles.

More recently, the first high-precision data from the KVI experiment at $190 \mathrm{MeV}$ became available [5], and many more data are forthcoming [6]. When comparing these data with theory, a pronounced and undisputable discrepancy between theory and experiment was observed in specific kinematic regions [5]. The size of the discrepancy between theory and experiment is disturbing, since what primarily enters are the two-nucleon $(N N)$ interaction and the electromagnetic coupling of the photon to the $N N$ system, both of which are believed to be accurately known at this energy. The high precision of the new KVI data allows one, in principle, to study smaller effects, similar to those arising from negative-energy states, the $\Delta$-isobar, and meson-exchange currents. It is therefore important to identify the possible reasons for the discrepancies.

In this paper, we compare the covariant model of Ref. [10] with the KVI data available so far and analyze the discrepancies. We demonstrate that the dominant contribution to $p p \gamma$ for the specific problematic kinematic regions results when the $N N$ interaction is evaluated at energies below about $15 \mathrm{MeV}$, and that, in fact, at least a major part of the problem resides in the low-energy behavior of the $N N$ interaction models used.

This paper is organized as follows. First, we review briefly the covariant model for $p p \gamma$ and compare it to the KVI data in the problematic kinematic regions. Next, we demonstrate the sensitivity of the bremsstrahlung cross sections in these regions to the properties of the low-energy $N N$ interaction. We then show that the problems can be significantly alleviated accordingly by improving the description of the interaction at low energies. We end by summarizing our conclusions.

\section{COVARIANT MODEL FOR BREMSSTRAHLUNG}

We first give a short review of the microscopic model of Martinus et al. for $p p \gamma$ [10]. In this covariant model the $N N$ $T$ matrix is obtained by solving the Bethe-Salpeter equation for the two-nucleon system [15] in the equal-time approximation with the one-boson exchange (OBE) kernel of Ref. [16]. This OBE model for the $N N$ interaction was, at that time, fitted to the Virginia Tech $n p$ partial-wave solution [17], by adjusting the meson-nucleon coupling constants and the form factor parameters, and a reasonable agreement was obtained.

This covariant $N N T$-matrix enters the model for $p p \gamma$, in which a number of contributions can be distinguished. The most important ones in the energy regime we consider here are the "nucleonic" contributions, consisting of singlescattering terms, i.e., photon emission off the external proton legs [see Figs. 1(a), 1(b)], and the contribution commonly known as rescattering [see Fig. 1(c)]. The model is relativistic covariant and therefore negative-energy states are included in a natural way. The relevance of these negativeenergy states is small for energies around $200 \mathrm{MeV}$. The

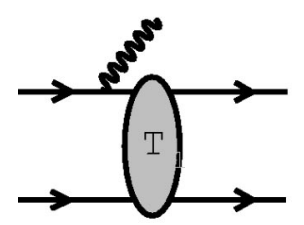

a)

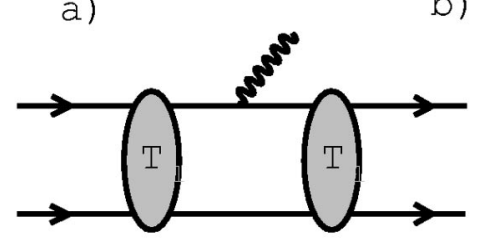

C)

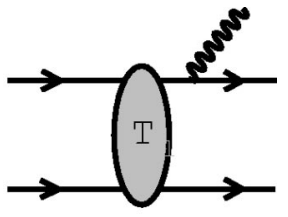

b)
FIG. 1. Single scattering (a), (b), and rescattering (c) contributions to $p p \gamma$. Analogous diagrams in which the lower proton radiates the photon are not shown. 


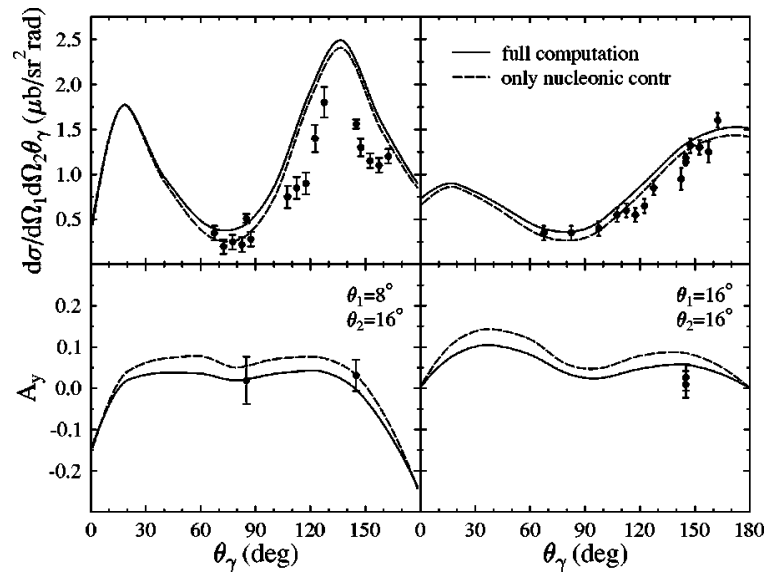

FIG. 2. Bremsstrahlung cross sections (upper panels) and analyzing powers (lower panels) at $190 \mathrm{MeV}$ incoming proton energy, and for proton angles $8^{\circ}, 16^{\circ}$ (left panels) and $16^{\circ}, 16^{\circ}$ (right panels). The solid curves show the results of the full model, including negative-energy states and two-body currents, while the nucleonic contribution is shown by the dashed lines. The KVI data (partly preliminary) are taken from Ref. [5].

reason [10] is that the contributions from the singlescattering diagrams where the intermediate nucleons are in a negative-energy state are canceled by similar contributions coming from the rescattering diagram. This cancellation holds for terms up to order $\mathcal{O}(q)$, where $q$ is the photon momentum, and is a consequence of the soft-photon theorem [18].

Contributions from the $\Delta$ isobar and from magnetic meson-exchange currents, containing in particular the $\omega \pi \gamma$ and $\rho \pi \gamma$ decay graphs, are also taken into account. These two-body current terms are included in a perturbative way, since they are small in general. The coupling constants of the photon to the various mesons were determined from the radiative decay widths of the vector mesons. As one can observe from Fig. 2 below, at an energy of $190 \mathrm{MeV}$ the contribution of the two-body currents is small. These terms, however, increase in size with energy and can become appreciable around the pion-production threshold at $280 \mathrm{MeV}$ and above.

This covariant bremsstrahlung model [10] is theoretically well founded and many of its ingredients have been tested in other calculations such as those for electron scattering on the deuteron [16]. Therefore, one did not expect major discrepancies with new experimental $p p \gamma$ data at energies below $280 \mathrm{MeV}$.

\section{COMPARISON WITH THE KVI DATA}

In the KVI experiment, $p p \gamma$ cross sections and analyzing powers were measured for $190 \mathrm{MeV}$ incoming proton energy, with the scattered protons detected at small forward angles, and with the photon emitted in the backward hemisphere [5]. A typical example of the KVI data [5] in comparison with theory is shown is Fig. 2. The data are plotted for two different kinematic situations, with $\theta_{1}$ and $\theta_{2}$ the fixed angles of the outgoing protons in the laboratory frame,

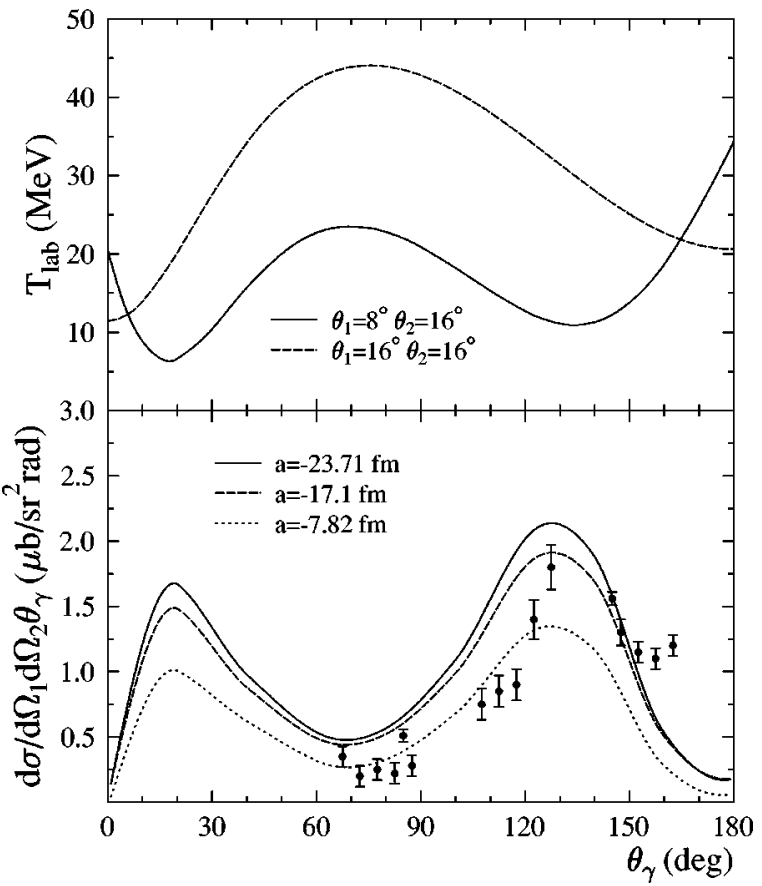

FIG. 3. Upper panel: kinetic energy of the incoming proton at which the $N N T$ matrix is evaluated for the two kinematics discussed in the text and in Fig. 2. Lower panel: bremsstrahlung cross sections at $190 \mathrm{MeV}$ for three different scattering lengths for $\theta_{1}$ $=8^{\circ}, \theta_{2}=16^{\circ}$. Only the contribution from the ${ }^{1} S_{0}$ partial wave is included.

and as function of the polar angle of emission of the photon $\theta_{\gamma}$. The theoretical predictions are from the model as published in Ref. [10].

For the asymmetric proton angles $\theta_{1}=8^{\circ}, \theta_{2}=16^{\circ}$ (upper left panel) the cross section shows a large discrepancy between theory and experiment for values of $\theta_{\gamma}$ corresponding to the backward peak in the cross section. For the symmetric proton angles $\theta_{1}=\theta_{2}=16^{\circ}$ (upper right panel), on the other hand, the cross section shows a much better agreement between theory and data. The contribution of the two-body currents is seen to be minor, and thus the discrepancy for $\theta_{1}=8^{\circ}, \theta_{2}=16^{\circ}$ is unlikely to come from this source. In the following, we focus therefore on the nucleonic contribution as the cause of the problem.

Taking a closer look at the two kinematics presented in Fig. 2 reveals that the one which poses problems $\left(\theta_{1}\right.$ $=8^{\circ}, \theta_{2}=16^{\circ}$ ) is dominated by the contribution from the ${ }^{1} S_{0}$ wave in the $N N T$ matrix, while for the second one for which the agreement is much better $\left(\theta_{1}=\theta_{2}=16^{\circ}\right)$ the ${ }^{3} P$ waves are as important as the ${ }^{1} S_{0}$ wave. The contribution of the rescattering diagram is relatively small, suggesting that the problem resides already at the level of the singlescattering diagrams.

In the bremsstrahlung calculation the $N N T$ matrix is evaluated at different energies for each of the diagrams shown in Fig. 1. The energy is lowest for the diagrams where the photon is emitted by one of the incoming protons. The value of this energy is shown in Fig. 3 as a function of $\theta_{\gamma}$. For $\theta_{1}=8^{\circ}, \theta_{2}=16^{\circ}$, the $T$ matrix is evaluated at 7 and 11 
TABLE I. Cross sections, in $\mu \mathrm{b} / \mathrm{sr}^{2} \mathrm{rad}$, for different kinematics $\theta_{1}, \theta_{2}, \theta_{\gamma}$, split up in partial waves, radiation from initial and final proton legs, and the total. For each kinematics, the first column gives the value of the original fit [10], while the second column gives the corresponding value after the refit.

\begin{tabular}{lcccc}
\hline \hline & $\theta_{1}, \theta_{2}, \theta_{\gamma}=8^{\circ}, 16^{\circ}, 140^{\circ}$ & $\theta_{1}, \theta_{2}, \theta_{\gamma}=16^{\circ}, 16^{\circ}, 160^{\circ}$ \\
\hline${ }^{1} S_{0}$ & 1.90 & 1.18 & 0.39 & 0.20 \\
${ }^{3} P_{0}$ & 0.02 & 0.02 & 0.01 & 0.02 \\
${ }^{3} P_{1}$ & 0.85 & 0.53 & 0.82 & 0.51 \\
${ }^{3} P_{2}$ & 0.43 & 0.42 & 0.53 & 0.47 \\
& & & & \\
initial & 2.31 & 1.53 & 0.80 & 0.55 \\
final & 0.93 & 0.68 & 0.98 & 0.76 \\
& & & & 1.38 \\
total & 2.40 & 1.98 & 1.44 & \\
\hline \hline
\end{tabular}

$\mathrm{MeV}$ kinetic energy for the cases corresponding to the peaks in the bremsstrahlung cross sections, i.e., at about $\theta_{\gamma}=20^{\circ}$ and $140^{\circ}$, respectively. For the minimum in the cross section, around $\theta_{\gamma}=75^{\circ}$, the $T$ matrix is evaluated at $24 \mathrm{MeV}$ when the photon is emitted by one of the incoming protons. In contrast, one observes that for $\theta_{1}=\theta_{2}=16^{\circ}$, the $T$ matrix is evaluated at more than $20 \mathrm{MeV}$ for $\theta_{\gamma}=160^{\circ}$ and at more than $40 \mathrm{MeV}$ for the minimum around $\theta_{\gamma}=75^{\circ}$; see the upper panel of Fig. 3. Thus, we conclude that theory and experiment agree well for those situations where the $T$ matrix is evaluated above about $15 \mathrm{MeV}$, while the discrepancies occur for the cases corresponding to a lower energy.

In order to demonstrate the sensitivity of the $p p \gamma$ cross section to the low-energy $N N$ interaction, we plot in the lower panel of Fig. 3 the cross section for $\theta_{1}=8^{\circ}, \theta_{2}$ $=16^{\circ}$, where we only include the ${ }^{1} S_{0}$ wave, for three values of the scattering length. Here we simply changed, for illustrative purpose only, the value of $g_{\varepsilon}^{2}$, the coupling constant of the " $\varepsilon$ meson" in the OBE model.

In Table I we list for the cases $\theta_{1}=8^{\circ}, \theta_{2}=16^{\circ}$, and $\theta_{1}$ $=\theta_{2}=16^{\circ}$, the cross sections calculated by including in the $N N T$ matrix only one of the important partial waves, viz. the ${ }^{1} S_{0}$ wave and the ${ }^{3} P$ waves. This is done for the value of $\theta_{\gamma}$ corresponding to the backward peak or plateau in the cross section, i.e., $\theta_{\gamma}=140^{\circ}$ in the first case and $\theta_{\gamma}=160^{\circ}$ in the second case, respectively. In both cases the first number listed gives the cross sections with the $T$ matrix as used in Ref. [10] (the second number gives the corresponding value in the fit described below). It is seen that the contribution of the ${ }^{1} S_{0}$ wave is significantly larger for the case $\theta_{1}=8^{\circ}, \theta_{2}$ $=16^{\circ}$ compared to the case $\theta_{1}=\theta_{2}=16^{\circ}$. By far the largest contribution to this ${ }^{1} S_{0}$ cross section comes from radiation off the incoming proton legs. The ${ }^{3} P$ wave cross sections arise mainly from radiation off the outgoing proton legs. For $\theta_{1}=8^{\circ}, \theta_{2}=16^{\circ}$ emission from the incoming proton legs dominates the bremsstrahlung cross section, while for $\theta_{1}$ $=\theta_{2}=16^{\circ}$ emission from initial and final proton legs are comparable. The total cross sections listed in the bottom row of Table I include also the rescattering contribution.

At the time of its construction, the OBE model of Ref.

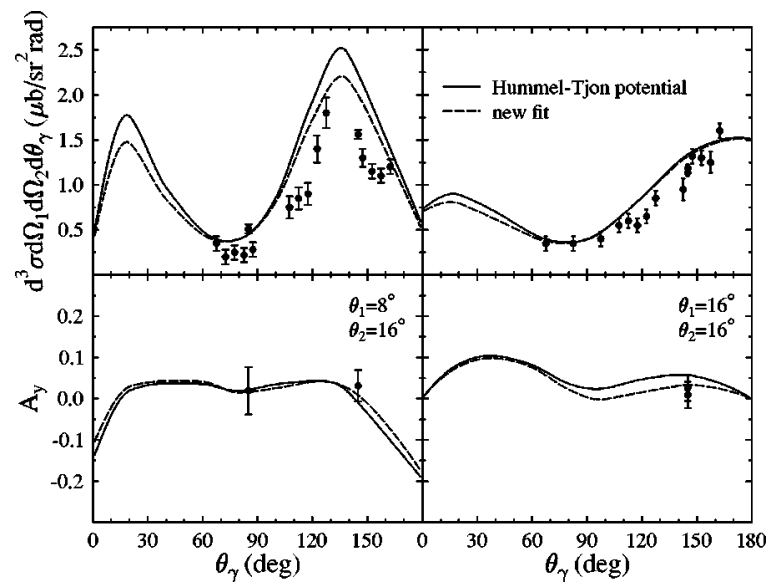

FIG. 4. Bremsstrahlung cross sections and analyzing powers at $190 \mathrm{MeV}$, for the same kinematics as in Fig. 2. The solid (dashed) curves show the results before (after) the refit of the $N N$ model.

[16] was fitted to the $n p$ phase-shift parameters of the Virginia Tech group, where the interest of the fit was at the higher energies. In view of our findings with the new highprecision $p p \gamma$ data, it becomes important to improve the description at low energies. In order to investigate whether at least the major part of the discrepancy between theory and the KVI data can be removed, we have performed a preliminary refit with an emphasis of obtaining better agreement with the Nijmegen $p p$ partial-wave analysis of Ref. [19], at energies starting from 10 up to $215 \mathrm{MeV}$.

In Fig. 4 we present the same calculation as in Fig. 2, but now for the $N N$ model after the preliminary new fit. The description of the $p p \gamma$ cross sections in the region of the backward peak for $\theta_{1}=8^{\circ}, \theta_{2}=16^{\circ}$ has improved substantially. This is mainly due to the improvement in the description of the ${ }^{1} S_{0}$ wave at low energies. Also the description of the ${ }^{3} P$ partial waves has improved. In Table I we now again list for the two kinematical situations the contribution to the p $\gamma$ cross section of the different important partial waves. The new results should be compared to the values obtained with the model as published in Ref. [10], and also listed in Table I.

A preliminary investigation suggests that for those regions in phase space where similar problems occurred as demonstrated here for the case $\theta_{1}=8^{\circ}, \theta_{2}=16^{\circ}$, at least a large part of the problem is due to an inadequate description of the low-energy behavior of the $N N T$ matrix. Detailed results will be presented in Ref. [20].

Finally, it is worth pointing out that the sensitivity of the pp $\gamma$ cross sections to the low-energy $N N$ interaction raises the question about the comparison between microscopic bremsstrahlung models of the type discussed here and softphoton descriptions. The soft-photon amplitude of Ref. [11] gives a remarkably good description of the experimental $p p \gamma$ cross sections, including the data from KVI. In constructing the soft-photon amplitude, a specific choice is made for the on-shell points used in the calculation. For the kinematic situations discussed here, this choice does not correspond to the $N N$ interaction at low energies, and therefore, the relation to microscopic models is not obvious. 


\section{CONCLUSIONS}

In conclusion, the $p p \gamma$ cross section at $190 \mathrm{MeV}$ varies strongly as a function of the angles of the protons. This can be traced back to an increasing contribution of the ${ }^{1} S_{0}$ wave for the regions in phase space where the $N N$ interaction is probed at low energies. For $\theta_{1}=8^{\circ}, \theta_{2}=16^{\circ}$, the ${ }^{1} S_{0}$ wave dominates strongly at the peak of the cross section. In contrast, for $\theta_{1}=\theta_{2}=16^{\circ}$, the ${ }^{3} P$ waves are as important as the ${ }^{1} S_{0}$ wave.

The present study shows that the predicted cross sections in certain kinematic regions are sensitive to the $N N$ interaction at low energy. In all cases, the analyzing powers are affected less by changes in the interaction. We have in particular not included in these calculations the Coulomb inter-

[1] K. Michaelian et al., Phys. Rev. D 41, 2689 (1990).

[2] R. Bilger et al., Phys. Lett. B 429, 195 (1998).

[3] J. Zlomanczuk, A. Johansson, and the WASA-PROMICE Collaboration, Nucl. Phys. A631, 622c (1998).

[4] K. Yasuda et al., Phys. Rev. Lett. 82, 4775 (1999).

[5] N. Kalantar-Nayestanaki et al., Nucl. Phys. A631, 242c (1998); H. Huisman et al., Phys. Rev. Lett. 83, 4017 (1999); Phys. Lett. B 476, 9 (2000).

[6] H. Huisman et al. (unpublished).

[7] V.R. Brown, P.L. Anthony, and J. Franklin, Phys. Rev. C 44, 1296 (1991).

[8] V. Herrmann and K. Nakayama, Phys. Rev. C 46, 2199 (1992); F. de Jong et al., Phys. Lett. B 333, 1 (1994); F. de Jong, K. Nakayama, and T.S.H. Lee, Phys. Rev. C 51, 2334 (1995); V. Herrmann et al., Nucl. Phys. A582, 568 (1995).

[9] J.A. Eden and M. Gari, Phys. Lett. B 347, 187 (1995); Phys. Rev. C 53, 1102 (1996).

[10] G.H. Martinus, O. Scholten, and J.A. Tjon, Phys. Lett. B 402, 7 (1997); Phys. Rev. C 56, 2945 (1997); 58, 686 (1998); FewBody Syst. 26, 197 (1999); G. H. Martinus, Ph.D. thesis, University of Groningen, 1998. action, which plays an important role in the $p p$ system at low energies. The results of such an analysis will be given in Ref. [20]. Only a limited set of KVI data has been published so far [5]. With the complete $p p \gamma$ data set [6], one may hope to be able to test in a quantitative way the validity of the various microscopic bremsstrahlung models.

\section{ACKNOWLEDGMENTS}

We acknowledge discussions with our experimental colleagues at KVI. The research of R.G.E.T. was made possible by the Royal Netherlands Academy of Arts and Sciences. We thank M. C. M. Rentmeester for his help in setting up the codes for fitting the $N N$ model.

[11] M.K. Liou, R. Timmermans, and B.F. Gibson, Phys. Lett. B 345, 372 (1995); 355, 606(E) (1995); Phys. Rev. C 54, 1574 (1996).

[12] A.Yu. Korchin and O. Scholten, Nucl. Phys. A581, 493 (1995); A.Yu. Korchin, O. Scholten, and D. Van Neck, ibid. A602, 423 (1996).

[13] Y. Li, M.K. Liou, R. Timmermans, and B.F. Gibson, Phys. Rev. C 58, R1880 (1998).

[14] S. Kondratyuk, G. Martinus, and O. Scholten, Phys. Lett. B 418, 20 (1998).

[15] J. Fleischer and J.A. Tjon, Nucl. Phys. B84, 375 (1974); Phys. Rev. D 15, 2537 (1977); 21, 87 (1990).

[16] E. Hummel and J.A. Tjon, Phys. Rev. C 42, 423 (1990); 49, 21 (1994).

[17] R.A. Arndt, J.S. Hyslop III, and L.D. Roper, Phys. Rev. D 35, 128 (1987).

[18] F.E. Low, Phys. Rev. 110, 974 (1958).

[19] V.G.J. Stoks et al., Phys. Rev. C 48, 792 (1993); M.C.M. Rentmeester et al., Phys. Rev. Lett. 82, 4992 (1999).

[20] M.D. Cozma, O. Scholten, R.G.E. Timmermans, and J.A. Tjon (in preparation). 